BMJ

Open

Gastroenterology

\section{Modelling the benefits of an optimised treatment strategy for 5-ASA in mild-to- moderate ulcerative colitis}

To cite: Louis E, Paridaens $\mathrm{K}$, Al Awadhi S, et al. Modelling the benefits of an optimised treatment strategy for 5-ASA in mild-to-moderate ulcerative colitis. BMJ Open Gastro 2022;9:e000853. doi:10.1136/ bmjgast-2021-000853

\section{- Additional supplemental material is published online only. To view, please visit the journal online (http://dx.doi. org/10.1136/bmjgast-2021- 000853).}

Received 3 December 2021 Accepted 25 January 2022
Check for updates

(C) Author(s) (or their employer(s)) 2022. Re-use permitted under CC BY-NC. No commercial re-use. See rights and permissions. Published by BMJ.

For numbered affiliations see end of article.

Correspondence to Dr Kristine Paridaens: kristine.paridaens@ferring.com

\section{ABSTRACT}

Objectives 5 -aminosalicylate (mesalazine; 5-ASA) is an established first-line treatment for mild-to-moderate ulcerative colitis (UC). This study aimed to model the benefits of optimising 5-ASA therapy.

Methods A decision tree model followed 10000 newly diagnosed patients with mild-to-moderately active UC through induction and 1 year of maintenance treatment. Optimised treatment (maximising dose of 5-ASA and use of combined oral and rectal therapy before treatment escalation) was compared with standard treatment (standard doses of 5-ASA without optimisation). Modelled data were derived from published meta-analyses. The primary outcomes were patient numbers achieving and maintaining remission, with an analysis of treatment costs for each strategy conducted as a secondary outcome (using UK reference costs).

Results During induction, there was a $39 \%$ increase in patients achieving remission through the optimised pathway without requiring systemic steroids and/or biologics (6565 vs 4725 for standard). Potential steroidal/ biological adverse events avoided included: seven venous thromboembolisms and eight serious infections. Out of the 6565 patients entering maintenance following successful induction on 5-ASA, there was a $21 \%$ reduction in relapses when optimised (1830 vs 2311 for standard). This translated into 297 patients avoiding further systemic steroids and 214 biologics. Optimisation led to an average net saving of $£ 272$ per patient entering the model for the induction and maintenance of remission over 1 year. Conclusion Modelling suggests that optimising 5ASA therapy (both the inclusion of rectal 5-ASA into a combined oral and rectal regimen and maximisation of 5-ASA dose) has clinical and cost benefits that supports wider adoption in clinical practice.

\section{INTRODUCTION}

Ulcerative colitis (UC) is a chronic inflammatory condition of the intestinal mucosa that affects the rectum and a variable extent of the colon and is commonly characterised by relapses separated by periods of remission. ${ }^{1}$ It can have a substantial negative effect on patients' quality of life due to the burden of

\section{Summary box}

What is already known about this subject?

- The optimisation of 5-aminosalicylate (mesalazine; 5-ASA) therapy in ulcerative colitis has the potential to maximise the efficacy of this first-line treatment option for mild-to-moderate disease. Optimisation of 5-ASA refers to the use of combined oral plus rectal therapy and maximising oral doses. The benefits of an optimised approach have yet to be fully assessed.

What are the new findings?

- This study quantified the benefits of an optimised 5-ASA treatment pathway for both the induction and maintenance of remission over standard (nonoptimised) management using a decision tree model. Optimisation led to reduced use of systemic steroids and advanced therapies, thereby avoiding the potential for adverse events associated with their use, while maintaining efficacy outcomes.

How might it impact on clinical practice in the foreseeable future?

- The wider adoption of an optimised 5-ASA treatment pathway is likely to have a number of benefits for both patients and healthcare systems, by maximising patient outcomes while minimising exposure to unnecessary therapies.

invasive symptoms as well as the psychological impacts and detriments to family and professional life. ${ }^{2} \mathrm{UC}$ is commonly classified by both disease severity (mild, moderate or severe) and extent (proctitis, left sided or extensive). ${ }^{1}$ 5-aminosalicylate (mesalazine; 5-ASA) is a well-established treatment for mild-to-moderate UC that is well tolerated and is the primary first-line treatment recommended in all international guidelines. ${ }^{34}$-ASA is used both for the induction and maintenance of remission in mild-tomoderate UC. ${ }^{34}$ Systemic oral corticosteroids are typically recommended as the initial 
step-up therapy for patients whose disease activity is not adequately controlled with 5-ASA. ${ }^{35}$ Systemic steroids are effective therapies for $\mathrm{UC},{ }^{5}$ but are also associated with many adverse events (AEs), such as, venous thromboembolism (VTE) ${ }^{67}$ psychological disturbances,${ }^{8}{ }^{9}$ weight gain, myopathy and osteoporosis in the longer term. ${ }^{58}$ Furthermore, steroids do not prevent relapse. There is, therefore, both a rationale and desire to avoid systemic steroids unless they are clearly necessary in these patients.

These facts have led to the concept of optimisation of 5-ASA treatment before escalating to systemic steroids or other advanced therapies. Optimisation of treatment aims to ensure that the best outcomes for patients are achieved while avoiding the use of systemic steroids whenever possible. ${ }^{10}$ The main approaches used for optimisation of 5-ASA are the use of combined oral plus rectal therapy and maximising oral doses. ${ }^{10}$ A number of studies have shown that these approaches can increase the effectiveness of 5-ASA therapy. ${ }^{11-13}$ A further step in 5-ASA optimisation has been the use of once daily dosing, which has demonstrated equivalent efficacy to divided dosing regimens and can improve patient adherence to treatment. ${ }^{12}{ }^{13}$ When moving beyond 5-ASA, further steps can be taken to optimise corticosteroid therapy by reducing systemic steroid exposure in order to minimise the incidence of AEs. The most common approaches are to use topically applied steroids (ie, rectally administered steroids for proctitis), or the use of a topically active oral steroid with a high first-pass metabolism, such as budesonide Multi Matrix (MMX) (with MMX delivery technology providing targeted colonic delivery) or beclomethasone dipropionate. ${ }^{10}{ }^{14}$ Recommendations for the use of optimised 5-ASA and optimised oral steroids are included within major treatment guidelines. ${ }^{341516}$ When these treatment options are not sufficient to induce remission, oral prednisolone is the commonly recommended next treatment, ${ }^{3}{ }^{4}$ with a number of advanced therapy options for further treatment when necessary (including immunomodulators, tumour necrosis factor inhibitors (anti-TNFs) and vedolizumab). ${ }^{3}$ During remission of UC on 5-ASA maintenance therapy, an additional optimisation step is to monitor disease activity through objective markers including endoscopic disease activity, imaging modalities including intestinal ultrasound and biomarkers, such as faecal calprotectin (FC) levels which can act as a trigger for 5-ASA dose escalation, when necessary. ${ }^{10}$

While there is a strong rationale for optimisation and good supporting evidence for individual aspects of 5-ASA optimisation, ${ }^{17-22}$ there has been less investigation into the benefits of a fully optimised treatment pathway in mild-to-moderate UC. This study aimed to use published evidence to model the benefits of the dose optimisation strategy in both induction and maintenance of remission in patients with mild-to-moderately active UC.

\section{MATERIALS AND METHODS Model}

A decision tree model was constructed in Microsoft Excel that considered a population of 10000 newly diagnosed patients with mild-to-moderately active UC (figure 1). These patients were assumed to have a disease history comparable to the patient groups within the clinical data used as model inputs. Due to differences in treatment protocols, proctitis was modelled separately from

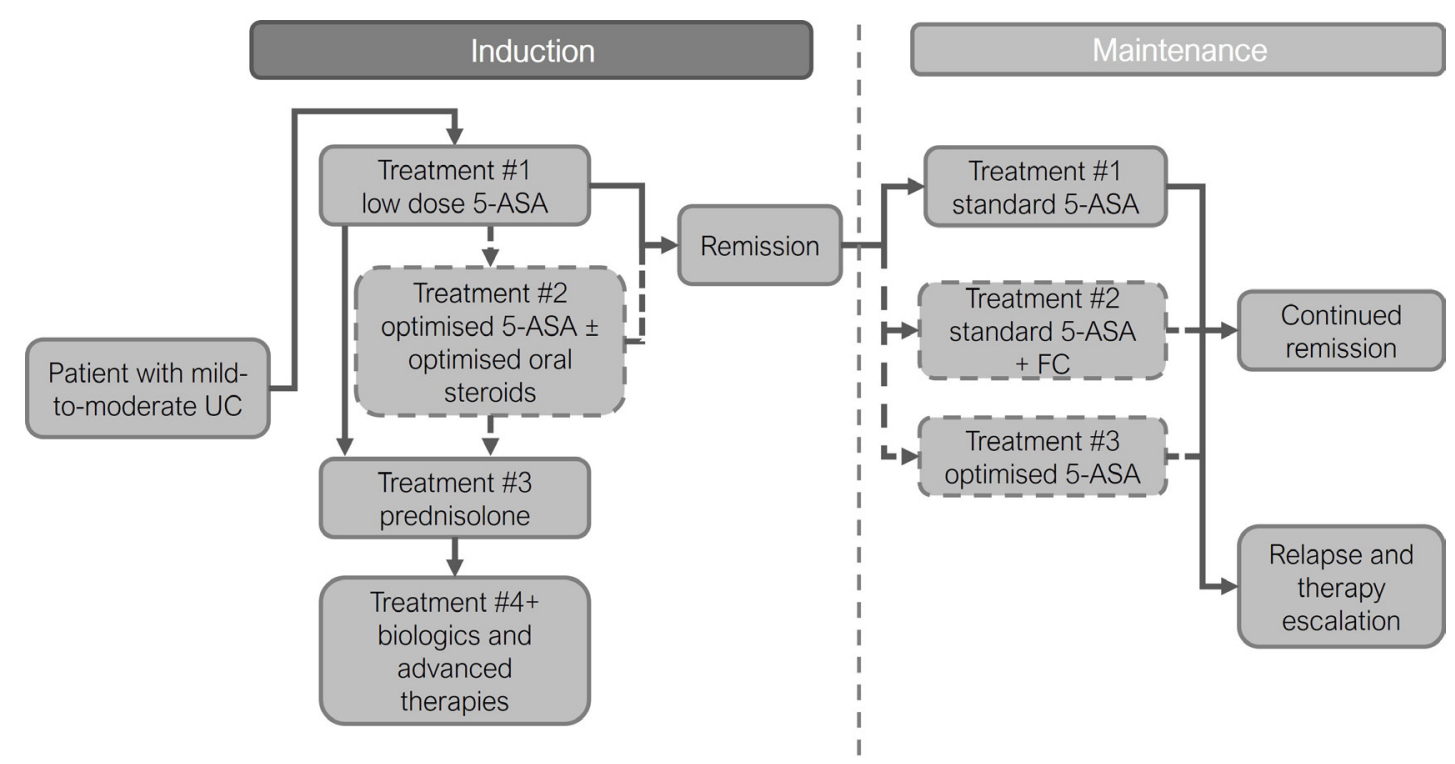

Figure 1 General model structure. Patients progress through model and treatments as indicated. The standard treatment pathway is marked by solid outlined boxes and arrows, additional treatment options for the optimised pathway are shown by dashed outlined boxes and arrows. In the induction phase, at the end of each treatment, patients reach a decision point where it is determined whether remission was achieved or the next induction treatment was required. In the maintenance phase, patients remain of one treatment for the whole 12-month period and the number remaining in remission at the end of this period is calculated. 5-ASA, 5-aminosalicylate; mesalazine; FC, faecal calprotectin; UC, ulcerative colitis. 
Table 1 Induction phase treatments

\begin{tabular}{llllll}
\hline \multirow{2}{*}{ Treatment no } & \multicolumn{2}{c}{ Proctitis } & & \multicolumn{2}{c}{ Left-sided and extensive } \\
\cline { 2 - 3 } \cline { 5 - 6 } & Standard & Optimised & & Standard & Optimised \\
\hline 1 & Rectal 5-ASA & Rectal 5-ASA & & Low-dose combined 5- & High-dose combined 5- \\
& Topical steroids or low- & High-dose combined & Oral prednisolone & Optimised oral steroids \\
3 & Oral steroids & Oral steroids & Biologics & Oral prednisolone \\
\hline 4 & Biologics & Biologics & & Biologics \\
\hline
\end{tabular}

5-ASA, 5-aminosalicylate; mesalazine.

left-sided and extensive disease, with $29.4 \%$ of the population modelled to have proctitis. ${ }^{23}$ The methods and inputs used in the base case (primary analysis) are described in the following sections.

For the induction phase of the model, patients progressed through the treatment pathway in a sequential manner with no repeated courses and no skipped treatments (pathway composed of 5-ASA treatments, steroid treatments and anti-TNF therapies, (table 1), with treatments based on clinical guideline recommendations. ${ }^{341516}$

After each treatment, a decision point was reached where it was assessed whether patients had achieved remission or remained with active disease. Once patients reached remission, they were not modelled to receive any further induction treatment; if patients remained with active disease then they progressed to the next treatment. Two induction treatment approaches were modelled: standard treatment (lower 5-ASA dose intensity (2-2.9 g/ day oral) ) and optimised treatment (including high-dose combined 5-ASA ( $\geq 3 \mathrm{~g}$ /day oral plus $1 \mathrm{~g}$ /day rectal) with/ without optimised oral steroids (using budesonide MMX as an example regimen)). All patients who achieved remission on optimised treatment without the need for systemic steroids (ie, achieved remission on 5-ASA, topical steroids or optimised oral steroids) were modelled to enter the 1-year maintenance phase; this was to ensure that the same number of patients in the standard and optimised pathways started maintenance therapy.

Within the maintenance phase, the standard treatment arm used a single low-dose 5-ASA treatment regimen for all patients (3-7g/week rectal for proctitis, or $2.4-3 \mathrm{~g}$ / day oral for left sided/extensive), whereas the optimised treatment arm used 5-ASA treatment options based on the dosing required for the induction of remission (rectal (3-7g/week), standard dose oral (2.4-3 g/day), high-dose oral (4-4.8g/day) or combined oral and rectal (2-8g/week rectal and 1.6-3 g/day oral) (table 2).

Patients in the maintenance phase were assumed to have reached the same degree of remission (equivalent to that seen in the clinical data) before starting maintenance. Within the optimised treatment pathway, FC levels exceeding $300 \mu \mathrm{g} / \mathrm{g}$ was considered as a trigger for the escalation of 5-ASA dose (from standard (2.4-3 g/day) to optimised (4-4.8g/day)), predicated on published evidence. ${ }^{24}$ At the end of the maintenance period, a decision point was reached where the relapse rate (as defined in 'model inputs' section) was used to calculate the number patients who remained on remission and the number who had relapsed.

\section{Model inputs}

Studies to inform the model were identified by targeted literature searches in MEDLINE, Embase and the Cochrane Library. Cochrane reviews were used as the primary data source, when available, as these were considered to provide the most robust and impartial evidence being well-conducted meta-analyses undertaken by a highly credible independent group. ${ }^{12} 25$ If no Cochrane review was available for a treatment, recent systematic literature reviews and meta-analyses were identified. ${ }^{111426}$ The non-Cochrane studies used provided sufficient detail

Table 2 Maintenance phase treatments

\begin{tabular}{|c|c|c|c|c|}
\hline \multirow[b]{2}{*}{ Treatment option } & \multicolumn{2}{|r|}{ Proctitis } & \multicolumn{2}{|c|}{ Left-sided and extensive } \\
\hline & Standard & Optimised & Standard & Optimised \\
\hline A & Rectal 5-ASA & $\begin{array}{l}\text { Rectal 5-ASA (following rectal 5-ASA } \\
\text { induction) }\end{array}$ & $\begin{array}{l}\text { Low-dose oral 5- } \\
\text { ASA }\end{array}$ & $\begin{array}{l}\text { Low-dose oral 5-ASA with FC } \\
\text { (following low-dose combined } \\
\text { 5-ASA induction) }\end{array}$ \\
\hline B & & $\begin{array}{l}\text { Low-dose oral 5-ASA with FC } \\
\text { (following topical steroids or low- } \\
\text { dose combined 5-ASA induction) }\end{array}$ & & $\begin{array}{l}\text { High-dose oral 5-ASA } \\
\text { (following high-dose combined } \\
5 \text {-ASA induction) }\end{array}$ \\
\hline C & & $\begin{array}{l}\text { Combined 5-ASA (following high- } \\
\text { dose combined 5-ASA induction) }\end{array}$ & & $\begin{array}{l}\text { Combined 5-ASA (following } \\
\text { budesonide MMX induction) }\end{array}$ \\
\hline
\end{tabular}

5-ASA, 5-aminosalicylate; mesalazine; FC, faecal calprotectin; MMX, Multi Matrix. 
on dosing to allow for the required dosing options in this model, and the reporting of clinical and/or endoscopic remission/relapse measures. If multiple studies fulfilled these criteria, the most recent and appropriate study was chosen to maximise the clinical data considered. Clinical remission (defined as per the included studies) was the chosen outcome in the induction phase as this was available for all modelled treatments. ${ }^{12142526}$ A pooled definition of relapse (using definitions of clinical and/or endoscopic relapse) and a 1-year time horizon was used for the maintenance part of the model. ${ }^{11}$ Full details on efficacy inputs are included as online supplemental tables 1 and 2.

A cost analysis was undertaken as a secondary outcome modelled from a UK perspective, due to the ready availability of published reference costs. Treatment acquisition costs were taken from the British National Formulary, using National Health Service (NHS) indicative prices (for generics/biosimilars, where available). ${ }^{27}$ Costs for administration were taken from NHS reference costs or Personal Social Services Research Unit data. ${ }^{28}{ }^{29}$ And costs for home FC testing (conducted every 3 months) were taken from those published by the National Institute for Health and Care Excellence. ${ }^{30}$ Full details on costs inputs are included as online supplemental tables $3-5$. An analysis of AE rates was also undertaken. AE rates were derived from published studies of the therapies of interest in a UC population, with preferred sources being the registration studies for biologic therapies and studies focused on the short-term AEs related to systemic steroids. $^{831-34}$

\section{Model outcomes}

For the induction part of the model, remission was the primary model outcome. The number of patients achieving remission was compared between the standard and optimised arms as the primary outcome. In addition, any differences in the requirements for systemic steroid treatment and biologics (anti-TNFs) were calculated. Results were presented as absolute and relative differences, with whole numbers presented for direct model outputs and proportions (or percentage differences) presented as percentages rounded to nearest integer (for simplicity and to avoid spurious accuracy). The treatment cost differences between the two arms were calculated as a secondary outcome (including only treatment acquisition and administration costs). Cost data are presented to the nearest whole pound. Potential AE differences between arms were calculated for systemic steroids and anti-TNFs.

For the maintenance part of the model, remission and relapse rates were compared between the two arms as the primary outcome. Excess relapses for standard over optimised maintenance treatment were modelled to re-enter induction, starting with systemic steroids and, if necessary, anti-TNF treatment. Treatment cost differences between the two arms was calculated as a secondary outcome (including only treatment acquisition and administration costs).

\section{Sensitivity and scenario analyses}

A one-way deterministic sensitivity analysis was conducted to show the sensitivity of the model to the main efficacy inputs. In this analysis, all efficacy inputs for both induction and maintenance phases were varied by a fixed amount of $\pm 20 \%$.

Additional scenario analyses were conducted to investigate other key model inputs. For the induction phase, a scenario was considered using a remission definition of combined clinical and endoscopic remission. This scenario used all available data for this composite remission definition, ${ }^{11} 1225$ with the base case (the principal analysis conducted) data used where no data for this endpoint was available (online supplemental table 1). ${ }^{1426}$ A further additional scenario explored the exclusion of budesonide MMX from the optimised pathway for leftside/extensive disease. For the maintenance phase, a scenario was considered where FC monitoring was not used and patients remained on a fixed dose of 5-ASA for the whole maintenance period. Finally, for the cost analyses, an illustrative scenario was conducted where $50 \%$ of patients who failed to achieve remission on systemic steroids were assumed to receive 6-mercaptopurine, azathioprine, tacrolimus or ciclosporin (the base case assumed that all these received anti-TNF therapy), to reflect clinical practice in countries where these therapies would be used as an alternative to anti-TNF therapy. Alternative biologic and advanced treatments were not considered here, but more expensive treatments such as vedolizumab and tofacitinib may also be used for this patient group in some countries, although anti-TNFs were considered the more widely used option by the authors.

\section{RESULTS}

\section{Induction}

For the 10000 patients entering the model, 4725 patients $(47 \%)$ achieved remission on standard dose 5-ASA compared with 6565 patients $(66 \%)$ on optimised 5-ASA, a relative increase of $39 \%$ for the latter (figure 2A). This led to 1840 patients $(18 \%)$ avoiding systemic steroids due to the use of the optimised 5-ASA regimen (3435 vs 5275 , respectively; $35 \%$ relative reduction in steroid use). When assessing use of anti-TNF therapies for those failing on systemic steroids, 1323 patients (13\%) avoided biological therapy in the optimised vs standard 5-ASA pathway (2469 vs 3792 patients, respectively; $35 \%$ relative reduction).

An analysis of AEs (related to escalation to systemic steroids such as prednisolone) showed that seven VTE events (95\% CI 6 to 7) along with 92 psychological AEs (95\% CI 53 to 132, effects such as steroid psychosis, mood disturbances, appetite changes and sleep disturbances), 239 gastrointestinal AEs (95\% CI 167 to 311), 
A

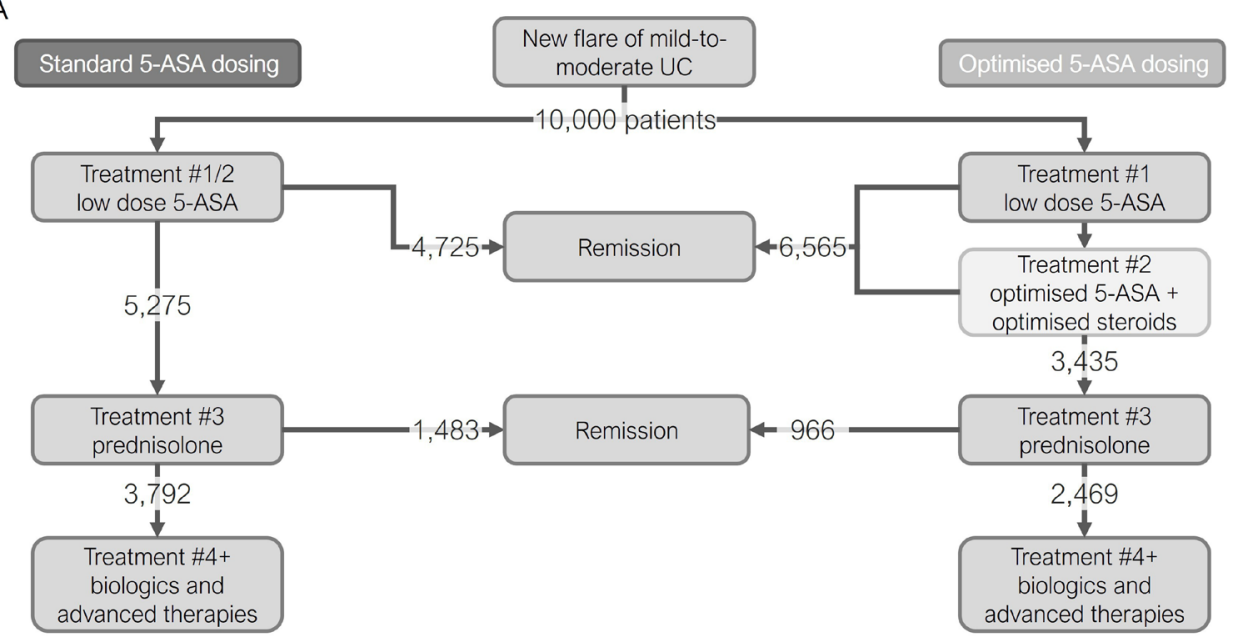

B

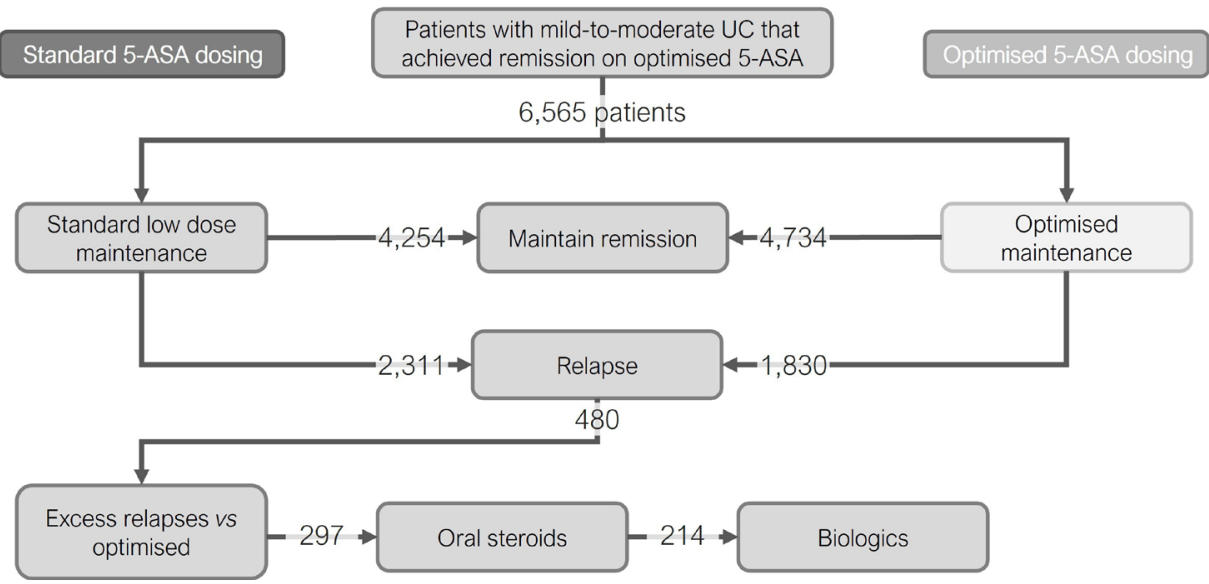

Figure 2 Induction and maintenance phase results. Flow diagram showing how patient numbers progress through key points of the induction (A) and maintenance (B) phases. 5-ASA, 5-aminosalicylate; mesalazine; UC, ulcerative colitis.

151 dermatological AEs (95\% CI 92 to 209), 198 neurological AEs (95\% CI 103 to 293, effects, such as headache, vertigo, dizziness and tinnitus) and 82 infection AEs (95\% CI 30 to 134) were potentially avoided by the reduced exposure to systemic steroids in the optimised arm. When considering anti-TNFs, the following AEs were potentially avoided by optimisation: 44 (95\% CI 18 to 70 , if treated with golimumab) or 83 (95\% CI 53 to 112 , if treated with adalimumab) injection-related reactions; 151 infusion-related reactions (95\% CI 73 to 233, if treated with infliximab); and 4 (95\% CI 0 to 12 , golimumab) or 8 (95\% CI 0 to 18 , adalimumab) serious infections (no relevant data on serious infections was available for infliximab). Additional data available for golimumab showed 40 headaches (95\% CI 15 to 65 ), 44 cases of nasopharyngitis (95\% CI 18 to 70 ) and 36 anaemia episodes (95\% CI 12 to 59 ) could potentially be avoided.

\section{Maintenance}

There were 6565 patients who entered the maintenance phase of the model (those achieving remission on optimised 5-ASA). During maintenance, 2311 patients experienced relapse with standard maintenance (effective relapse rate of $35 \%$ ), whereas 1830 patients experienced relapse with optimised maintenance (effective relapse rate of $28 \%$ ). There were, therefore, 480 patients who avoided relapse due to the optimised maintenance regimen, which is an improvement of $21 \%$ compared with standard maintenance (figure 2B). If these excess relapses were returned to the standard induction pathway again this would lead to an additional 297 patients requiring oral steroids ( $5 \%$ of maintenance population) and 214 patients requiring anti-TNF therapy (3\% of maintenance population); these were avoided through the use of the optimised maintenance regimen.

\section{Costs}

In the induction phase, the acquisition and administration costs of anti-TNF and systemic steroids avoided were $£ 5.4 \mathrm{~m}$ which were partially offset by the additional cost of treatment in the optimised 5-ASA treatment pathway $(£ 1.8 \mathrm{~m})$. Taken together, this led to a net cost saving for the optimised 5-ASA pathway of $£ 3.5 \mathrm{~m}$ over standard 5-ASA therapy in the induction of remission. This is equivalent to an average net saving of $£ 354$ per patient from optimised 5-ASA therapy.

In the maintenance phase, there were additional costs from the optimised treatment strategy of $£ 1.7 \mathrm{~m}$. 


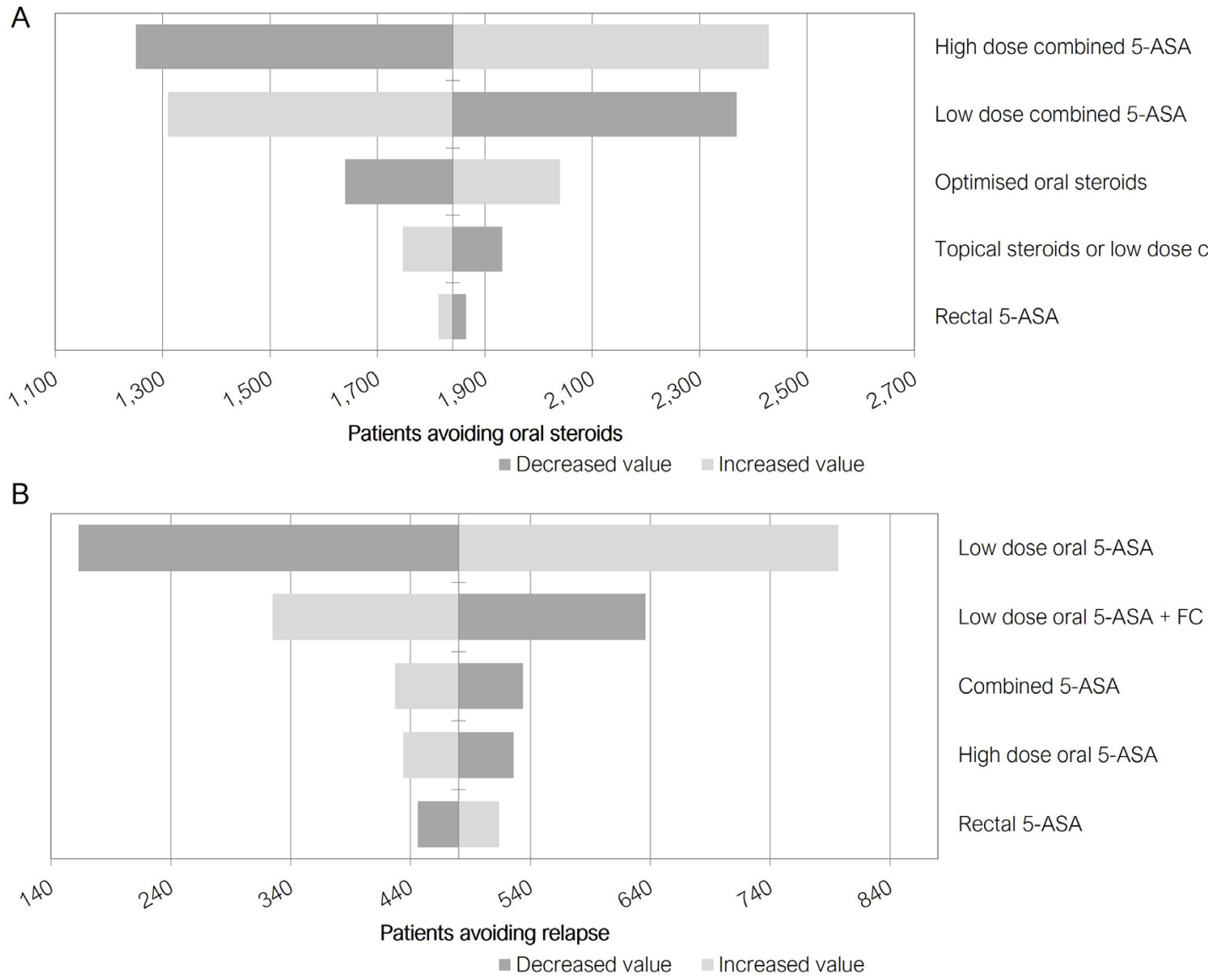

Figure 3 One way sensitivity analyses results. Tornado diagrams showing impact of varying efficacy inputs by $\pm 20 \%$ in induction (A) and maintenance (B) phases. As main model outcomes are clinical, these are presented as number of patients avoiding oral steroids (induction), (A) or number of patients avoiding relapse (maintenance), (B). Some inputs act in opposite directions on the model outputs when they are primarily associated to opposing arms of the model. 5-ASA, 5-aminosalicylate; mesalazine; FC, faecal calprotectin.

However, this was partially offset by the additional costs of reintroducing induction therapy (starting with systemic steroids) for the patients experiencing a relapse in the standard maintenance arm, which totalled $£ 1.0 \mathrm{~m}$. Altogether, there was a net additional cost of treatment (acquisition and administration) for the optimised arm of $£ 0.8 \mathrm{~m}$, equivalent to an average cost of $£ 125$ per patient entering the maintenance phase of the model.

When these two treatment phases are combined, the model shows a net cost saving for the optimised treatment strategy of $£ 2.7 \mathrm{~m}$. This works out to be an average saving of $£ 272$ per patient entering the model for the induction and maintenance of remission over 1 year. Full results of this cost analysis are included in online supplemental table 6 .

\section{Scenario and sensitivity analyses}

The one-way sensitivity analysis results (figure 3A) show that in the induction phase, high-dose combined 5-ASA and low-dose combined 5-ASA efficacy were the inputs that have the greatest impact on results. These inputs acted in opposite directions on the model as they are primarily used in opposing arms of the model. Under this sensitivity analysis the patients who avoid systemic steroids reached a minimum of 1250 and a maximum of 2429 (base case 1840), showing a substantial benefit to the optimised treatment pathway under a range of efficacy inputs. For the maintenance phase, (figure 3B) low-dose oral and low-dose oral with FC monitoring were the inputs that have the greatest impact on results. Again, these inputs acted in opposite directions on the model as they are primarily used in opposing arms of the model. Under this sensitivity analysis, the patients who avoided relapse reached a minimum of 163 and a maximum of 797 (base case 480) under the optimised treatment pathway relative to standard dosing.

When a definition of combined remission (patients achieving both clinical and endoscopic remission) was used as an alternative scenario in the induction phase, there were 1140 patients who would avoid systemic steroids and 819 who would avoid biologics (vs the base case of 1840 and 1323 , respectively). This analysis was limited in that where no data were available for combined remission for a treatment, the base case inputs were used. The scenario excluding the use of optimised oral steroids in left-sided/extensive disease led to 841 patients avoiding oral steroids and 604 avoiding 
anti-TNF therapy during induction. In the analysis where FC was not used within the maintenance phase (fixed dose of 5-ASA), 160 relapses would be avoided, which compared with 480 in the base case. This equates to the potential for 320 relapses to be prevented when FC monitoring is used to guide 5-ASA dose escalation rather than patients remaining on standard dose 5-ASA. The final scenario analysis that used a mix of therapies after systemic steroids found that in induction this led to an additional cost of treatment of $£ 3.0 \mathrm{~m}$ (compared with $£ 5.4 \mathrm{~m}$ in the base case). Under this scenario, there was a net cost saving for the optimised 5-ASA pathway of $£ 1.2 \mathrm{~m}$, equivalent to an average net saving of $£ 119$ per patient (compared with $£ 359$ per patient in the base case). For the maintenance phase, the reinduction costs were $£ 0.6 \mathrm{~m}$ in this scenario (compared with $£ 1.0 \mathrm{~m}$ in the base case). This led to a net additional cost for the optimised arm of $£ 1.2 \mathrm{~m}$, equivalent to an average cost of $£ 183$ per patient (compared with $£ 124$ per patient in the base case). This scenario highlights that costs are strongly influenced by the costs of advanced therapies used after systemic steroid failure in these patients. It also highlights that even under this scenario with reduced costs for advanced therapies, the optimised pathway has cost savings in the induction phase that match the increased costs in the maintenance phase. For the overall costs of both treatment phases, these scenario analyses demonstrate a range from an additional cost of £1.26 per patient when using a mix of therapies in place of anti-TNFs to a saving of £283 per patient when not using FC monitoring (a marginal increase in cost above the base case of $£ 277$ saving per patient).

\section{DISCUSSION}

The modelling conducted in this study indicates that an optimised treatment pathway for mild-to-moderate UC has clear benefits for patients and healthcare systems. The optimised pathway allowed a substantial additional number of patients (a relative increase of over a third (39\%)) to achieve remission without requiring systemic steroids (such as prednisolone) or advanced therapies (such as anti-TNFs). The modelling showed that the use of optimised 5-ASA and optimised oral steroids both contributed to achieving this benefit. The optimised pathway also allowed more patients to maintain this remission over 12 months (11\% increase in patients maintaining remission). The ability to achieve and maintain remission using optimised 5-ASA therapy and locally acting steroids has several key clinical benefits. First, these treatments are able to achieve remission rapidly and are generally well tolerated. ${ }^{34121325}$ It is well documented that systemic steroids and advanced therapies, such as antiTNFs, have the potential for serious AEs, ${ }^{5-8} 31-34$ which this study shows could be avoided in those achieving remission using an optimised approach. Almost 300 psychological and neurological AEs through exposure to systemic steroids (such as prednisolone) were avoided in the 10000 patients modelled. Second, optimisation allows the advanced therapies to be reserved until needed by maximising use of the current therapy before stepping up treatment—providing physicians more room for future therapeutic manoeuvre. This is particularly important when considering the welldocumented development of anti-drug antibodies with anti-TNFs. ${ }^{32-34}$ Finally, the benefits of optimisation, as demonstrated in the model, should be taken in the context of patient quality of life and other wider benefits associated with achieving and maintaining remission for patients with UC. These benefits are why the steps of optimised treatment are recognised as the current best standard of care and are recommended in treatment guidelines. ${ }^{34}$

This study also included an economic analysis based solely on the costs of treatment, which showed that there are potential cost savings in the induction phase (£354 per patient) and a small additional cost in the maintenance phase ( $\$ 125$ per patient) associated with the use of the optimised treatment pathway. Overall, these analyses imply that it is highly likely that implementing the optimised pathway would be cost beneficial (at the very least cost neutral) compared with the standard treatment pathway. The model shows an overall cost saving of $£ 272$ per patient entering the model. This analysis did not include additional healthcare costs associated with relapse, which were outside the scope and aim of the modelling. Additional costs that could be considered within full economic modelling would include costs related to medical practitioner time, diagnostic and additional procedures, hospital admissions, and with a wider perspective could also consider costs from lost productivity and impacts on carers time. A number of economic analyses have been previously published in this area, but these have only considered individual aspects of 5-ASA optimisation, including once daily dosing vs twice daily, ${ }^{17-20}$ combined 5 -ASA vs oral alone,${ }^{21}$ and high-dose oral 5-ASA vs low-dose oral 5-ASA. ${ }^{22}$ In all cases, these analyses showed that the optimised therapy was the dominant treatment option (lower costs and higher-quality-adjusted life-years). ${ }^{17-22} \mathrm{~A}$ further study focused on the costs and health-related quality of life (HRQoL) impacts of active UC and showed that there were high costs of care (direct and indirect) and adverse effects on HRQOL. ${ }^{35}$ Combined with the results from our modelling, it would suggest that the optimised treatment pathway is highly likely to be cost-saving and therefore likely to be dominant over the standard treatment pathway. This is a potential area for further investigation and future extension of this current model.

A key limitation of this model is that it represents a simplification of clinical reality, as it is assumed that patients follow the induction protocol in a strict sequential manner with no repeated courses or skipping of treatments. This decision was made to allow a clearer comparison and feasible model to be constructed. This means, however, that the model is not a perfect reflection of the clinical reality of treating patients with UC, and also that it cannot reflect variations in clinical practice between countries. The necessary simplifications have made a model that demonstrates the benefits of the optimised pathway in a slightly idealised situation. This does not detract from the results and the benefits shown for the optimised pathway but allows these benefits to be more clearly seen. Also, this approach and model allows 
the benefits of a wider optimised pathway to be considered when previous studies have only focused on the benefits of individual optimisation approaches. This, therefore, allows this study to show the substantial benefits from a fully optimised treatment pathway.

A further limitation is in the data available to use for construction of this model and the variation in definitions that have been used in trials of therapies in UC. ${ }^{11-13}$ This limited the definition of remission used in this model to clinical remission in the base case, when a definition of combined clinical and endoscopic remission would have been preferred as a more robust and clinically relevant definition. However, this is an issue that has been strongly influenced by the evolution in thinking and treatment aims over the years and is, therefore, applicable to any study aiming to pool data on the efficacy of 5-ASA. This is counteracted by the large number of studies that have been conducted, and continued to be conducted, into these treatments which provides a comprehensive evidence base. The inputs to the model were also robust in that they were derived from wellconducted meta-analysis by credible independent groups, such as Cochrane. While a number of studies have looked at individual aspects of optimisation, a more comprehensive assessment of this strategy is desirable. The current OPTIMISE study aims to provide additional evidence in this area, including a more personalised approach to therapy escalation and de-escalation. ${ }^{36}$ The OPTIMISE study should provide important new evidence in this area that complement the results from this model.

Another important factor in the efficacy of treatment is adherence to and persistence on therapy. This is particularly important during maintenance treatment where a longer duration of therapy is required in patients who are in remission and have no symptoms at that time. This model does not consider these two factors directly, but where available has used analyses based on an intention-to-treat approach, which therefore includes a consideration of patients who drop out and stop therapy. The model was not designed to consider the potential impact of adherence in a real-world setting, but maintenance phase therapies were the least onerous options to try and ensure that adherence would be maintained in a real-world setting. In this regard, the use of FC monitoring is particularly important as this allows patients to use a lower dose of 5-ASA and only escalate dosing as required. It should also be noted that this model used an FC threshold of $300 \mu \mathrm{g} / \mathrm{g}$ for dose escalation (predicated on the clinical study providing efficacy data for this approach), ${ }^{24}$ although other thresholds have been investigated for predicting relapse in UC. ${ }^{37}$ There could be arguments for using a lower FC threshold to ensure treatment escalation occurs in time to prevent relapse, but current evidence is inconclusive. The best approach is likely to be based on clinical judgement for the FC values of an individual patient (and their relative changes with treatment) to decide when treatment escalation should occur. Another advantage with the incorporation of FC monitoring should be that it helps to engage patients with their own treatment and, when combined with education to ensure that patients understand the requirements and importance of their therapy, should help to maintain adherence.

This study and modelling exercise highlights the potential clinical and cost benefits that can be achieved with an optimised treatment pathway. This includes a substantial number of patients avoiding the need for systemic steroids or other advanced therapies. Even without a full consideration of quality of life effects and all disease-related costs, the economic analysis conducted showed that the optimised pathway was likely to be cost saving (or at the very least cost neutral). The wider adoption of an optimised treatment pathway would likely have great benefits for both patients and healthcare systems.

\section{Author affiliations}

${ }^{1}$ Hepato-Gastroenterology and Digestive Oncology Department, University and Centre Hospitalier Univestitaire (CHU) Liège, Liège, Belgium

${ }^{2}$ Ferring International Center SA, Saint-Prex, Switzerland

${ }^{3}$ Gastroenterology Department, Rashid Hospital, Dubai, UAE

${ }^{4}$ Department of Gastroenterology, Mater Hospital Brisbane, Brisbane, Queensland, Australia

${ }^{5}$ Department of Internal Medicine, Yonsei University College of Medicine, Seoul, Korea (the Republic of)

${ }^{6}$ Department of Medicine I, Agaplesion Markus Hospital, Goethe-University, Frankfurt am Main, Germany

${ }^{7}$ Department of Biomedicine, Unit of Pharmacology and Therapeutics, University of Porto, Porto, Portugal

${ }^{8}$ Department of Gastroenterology, São João University Hospital, Porto, Portugal

${ }^{9}$ Colorectal Surgery Department, Instituto de Coloproctologia ICO Clinica Las Americas, Medellin, Colombia

${ }^{10}$ University Clinic for Internal Medicine, Johannes Kepler University, Linz, Austria

${ }^{11}$ Division of Gastroenterology, Department of Medicine and Farncombe Family

Digestive Health Research Institute, McMaster University, Hamilton, Ontario, Canada

${ }^{12}$ Clinical Department of Internal Medicine and Gastroenterology with Inflammatory Bowel Disease Subdivision, The Central Clinical Hospital of the Ministry of the Interior Affairs and Administration, Warsaw, Poland

${ }^{13}$ Collegium Medicum, Jan Kochanowski University of Kielce, Kielce, Poland

${ }^{14}$ Violicom Medical Limited, Aldermaston, UK

${ }^{15} \mathrm{NIHR}$ Oxford Biomedical Research Centre, Oxford University Hospitals NHS Foundation Trust, John Radcliffe Hospital, Oxford, UK

Acknowledgements Writing support was provided by Violicom Medical Limited and was funded by Ferring Pharmaceuticals.

Contributors All authors were responsible for conceptualisation, methodology, investigation, formal analysis, writing, manuscript review. All authors approved the final manuscript. MJF is the guarantor for this work.

Funding This work was supported by Ferring Pharmaceuticals.

Competing interests EL has received research grants from Janssen, Pfizer, and Takeda; educational grants from AbbVie, Janssen, MSD and Takeda; speaker fees from AbbVie, Falk, Ferring, Hospira, Janssen, MSD, Pfizer, and Takeda; participated in advisory boards for AbbVie, Celgene, Ferring, Hospira, Janssen, MSD, Pfizer, Takeda, Galapagos, Gilead, Arena, Elli Lilly; consultant for AbbVie. $\mathrm{KP}$ is an employee of Ferring Pharmaceuticals. SAA has participated in advisory board for Ferring. JB has received honoraria, research grants or consulting fees from Abbvie, Janssen, Takeda, Pfizer, Ferring, Bristol Myers Squibb, Gilead, Tillott's, Sandoz, Chiesi, Celltrion, Microba and Antara. JHC has received personal fees from Celltrion, Inc. Eisai Korea, Ferring Korea, IQVIA, Ferring, Janssen Korea, Shire Korea and Takeda Korea. AUD has received fees for participation in clinical trials, review activities, such as data monitoring boards, statistical analysis, end point committees from Falk, Abbvie, Janssen, Gilead and Pfizer; consultancy fees from Abbvie, MSD, Ferring, Roche/Genentech, Takeda, Vifor, Pharmacosmos, BoehringerIngelheim, Falk, Janssen, Pfizer, Sandoz/Hexal, BMS/ Celgene, Tillotts, Amgen and Fresenius Kabi; payment from lectures including service on speakers bureaus from Falk Foundation, Ferring, MSD, Abbvie, Vifor, Janssen, Pfizer, Tillotts, Takeda, Gilead/ Galapagos; payment for development of educational presentations from Tillotts and Ferring. FM has served as speaker and received honoraria from Abbvie, Biogen, 
Bristol-Myers Squibb, Falk, Ferring, Hospira, Janssen, Laboratórios Vitoria, Pfizer, Lilly, Merck Sharp \& Dohme, Sandoz, Takeda, UCB and Vifor. JRM has received sponsorship as speaker from AbbVie, Biopas, Biotoscana, Farma, Ferring, Janssen, and Takeda. ARM is receiving research support from AbbVie and Takeda under the framework of the Christian Doppler Research Society; has received further consultation fees and/or speaker honoraria from AbbVie, Merck Sharp \& Dohme, Takeda, Janssen-Cilag, Amgen, Sandoz, Nestlé, Ferring, Falk, and Pfizer. NN has received grants, advisory board fees, or speakers bureau honoraria from Janssen, Abbvie, Takeda, Pfizer, Merck, Sandoz, Novartis, and Ferring. GR has received grants/research support or speakers fee from Abbvie, AlfaSigma, Astellas, Ferring, Janssen, Pfizer, Pharmabest, Recordatti, Sanprobi, Sandoz, Vitama and Takeda. MJF is an employee of Violicom Medical Limited that has received funding from Ferring for work on various projects. SPLT has received Grants/Research Support from: AbbVie, Buhlmann, Celgene, IOIBD, Janssen, Lilly, Pfizer, Takeda, UCB, Vifor and Norman Collisson Foundation; Consulting Fees from: Abacus; AbbVie; Actial; ai4gi; Alcimed; Allergan; Amgen; Aptel; Arena; Asahi; Aspen; Astellas; Atlantic; AstraZeneca; Barco; Biocare; Biogen; BLPharma; Boehringer Ingelheim; BMS; Buhlmann; Calcico; Celgene; Cellerix; Cerimon; ChemoCentryx; Chiesi; CisBio; ComCast; Coronado; Cosmo; Ducentis; Dynavax; Elan; Enterome; Equillium; Falk; Ferring; FPRT Bio; Galapagos; Genentech/Roche; Genzyme; Gilead; Glenmark; Grunenthal; GSK; GW Pharmaceuticals; Immunocore; Immunometabolism; Indigo; Janssen; Lexicon; Lilly; Medarex; Medtrix; Merck; Merrimack; Millenium; Neovacs; Novartis; Novo Nordisk; NPS-Nycomed; Ocera; Optima; Origin; Otsuka; Palau; Pentax; Pfizer; Pharmaventure; Phillips; P\&G; Pronota; Proximagen; Resolute; Robarts; Sandoz; Santarus; Satisfai; Sensyne; Shire; SigmoidPharma; Sorriso; Souffinez; Syndermix; Synthon; Takeda; Theravance; Tigenix; Tillotts; Topivert; Trino Therapeutics with Wellcome Trust; TxCell; UCB Pharma; Vertex; VHsquared; Vifor; Warner Chilcott and Zeria; Speaker Fees from: AbbVie, Amgen, Biogen, Falk; Ferring, Janssen, Pfizer, Shire, Takeda, UCB; ST holds no stocks or share options.

Patient consent for publication Not applicable.

Ethics approval This study does not involve human participants.

Provenance and peer review Not commissioned; externally peer reviewed.

Data availability statement All data supporting the findings of this study are available within the article or online supplemental information and no additional source data are required.

Supplemental material This content has been supplied by the author(s). It has not been vetted by BMJ Publishing Group Limited (BMJ) and may not have been peer-reviewed. Any opinions or recommendations discussed are solely those of the author(s) and are not endorsed by BMJ. BMJ disclaims all liability and responsibility arising from any reliance placed on the content. Where the content includes any translated material, BMJ does not warrant the accuracy and reliability of the translations (including but not limited to local regulations, clinical guidelines, terminology, drug names and drug dosages), and is not responsible for any error and/or omissions arising from translation and adaptation or otherwise.

Open access This is an open access article distributed in accordance with the Creative Commons Attribution Non Commercial (CC BY-NC 4.0) license, which permits others to distribute, remix, adapt, build upon this work non-commercially, and license their derivative works on different terms, provided the original work is properly cited, appropriate credit is given, any changes made indicated, and the use is non-commercial. See: http://creativecommons.org/licenses/by-nc/4.0/.

ORCID iD

Matthew J Freddi http://orcid.org/0000-0001-8219-1135

\section{REFERENCES}

1 Magro F, Gionchetti P, Eliakim R, et al. Third European evidencebased consensus on diagnosis and management of ulcerative colitis. Part 1: definitions, diagnosis, extra-intestinal manifestations, pregnancy, cancer surveillance, surgery, and ileo-anal pouch disorders. J Crohns Colitis 2017;11:649-70.

2 Kobayashi T, Siegmund B, Le Berre C, et al. Ulcerative colitis. Nat Rev Dis Primers 2020;6:74.

3 Harbord M, Eliakim R, Bettenworth D, et al. Third European evidence-based consensus on diagnosis and management of ulcerative colitis. Part 2: current management. J Crohns Colitis 2017;11:769-84.

4 Ko CW, Singh S, Feuerstein JD, et al. AGA clinical practice guidelines on the management of mild-to-moderate ulcerative colitis. Gastroenterology 2019;156:748-64.
5 Lichtenstein GR, Abreu MT, Cohen R, et al. American gastroenterological association Institute technical review on corticosteroids, immunomodulators, and infliximab in inflammatory bowel disease. Gastroenterology 2006;130:940-87.

6 Isidori AM, Minnetti M, Sbardella E, et al. Mechanisms in endocrinology: the spectrum of haemostatic abnormalities in glucocorticoid excess and defect. Eur $J$ Endocrinol 2015;173:R101-13.

7 Johannesdottir SA, Horváth-Puhó E, Dekkers OM, et al. Use of glucocorticoids and risk of venous thromboembolism: a nationwide population-based case-control study. JAMA Intern Med 2013;173:743-52.

8 Hoes JN, Jacobs JWG, Verstappen SMM, et al. Adverse events of low- to medium-dose oral glucocorticoids in inflammatory diseases: a meta-analysis. Ann Rheum Dis 2009;68:1833-8.

9 Fietta P, Fietta P, Delsante G. Central nervous system effects of natural and synthetic glucocorticoids. Psychiatry Clin Neurosci 2009;63:613-22.

10 Solitano V, D'Amico F, Fiorino G, et al. Key strategies to optimize outcomes in mild-to-moderate ulcerative colitis. J Clin Med 2020;9:2905.

11 Barberio B, Segal JP, Quraishi MN, et al. Efficacy of oral, topical, or combined oral and topical 5-Aminosalicylates, in ulcerative colitis: systematic review and network meta-analysis. J Crohns Colitis 2021;15:1184-96.

12 Murray A, Nguyen TM, Parker CE, et al. Oral 5-aminosalicylic acid for induction of remission in ulcerative colitis. Cochrane Database Syst Rev 2020;8:CD000543.

13 Murray A, Nguyen TM, Parker CE, et al. Oral 5-aminosalicylic acid for maintenance of remission in ulcerative colitis. Cochrane Database Syst Rev 2020;8:CD000544.

14 Manguso F, Bennato R, Lombardi G, et al. Efficacy and safety of oral beclomethasone dipropionate in ulcerative colitis: a systematic review and meta-analysis. PLoS One 2016;11:e0166455.

15 Lamb CA, Kennedy NA, Raine T, et al. British Society of gastroenterology consensus guidelines on the management of inflammatory bowel disease in adults. Gut 2019;68:s1-106.

16 Bressler B, Marshall JK, Bernstein CN, et al. Clinical practice guidelines for the medical management of nonhospitalized ulcerative colitis: the Toronto consensus. Gastroenterology 2015;148:1035-58.

17 Connolly MP, Nielsen SK, Currie CJ, et al. An economic evaluation comparing once daily with twice daily mesalazine for maintaining remission based on results from a randomised controlled clinical trial. J Crohns Colitis 2009;3:32-7.

18 Connolly MP, Boersma C, Oldenburg B. The economics of mesalazine in active ulcerative colitis and maintenance in the Netherlands. Neth J Med 2012;70:272-7.

19 Connolly MP, Kuyvenhoven JP, Postma MJ, et al. Cost and qualityadjusted life year differences in the treatment of active ulcerative colitis using once-daily $4 \mathrm{G}$ or twice-daily $2 \mathrm{~g}$ mesalazine dosing. $J$ Crohns Colitis 2014;8:357-62.

20 Nishikawa AM, Paladini L, Delfini R, et al. Decision tree construction and cost-effectiveness analysis of treatment of ulcerative colitis with pentasa $\AA$ mesalazine $2 \mathrm{G}$ sachet. Arq Gastroenterol 2013;50:297-303.

21 Connolly MP, Nielsen SK, Currie CJ, et al. An economic evaluation comparing concomitant oral and topical mesalazine versus oral mesalazine alone in mild-to-moderately active ulcerative colitis based on results from randomised controlled trial. $J$ Crohns Colitis 2009;3:168-74.

22 Buckland A, Bodger K. The cost-utility of high dose oral mesalazine for moderately active ulcerative colitis. Aliment Pharmacol Ther 2008;28:1287-96.

23 Fumery M, Singh S, Dulai PS, et al. Natural history of adult ulcerative colitis in population-based cohorts: a systematic review. Clin Gastroenterol Hepatol 2018;16:343-56.

24 Lasson A, Öhman L, Stotzer P-O, et al. Pharmacological intervention based on fecal calprotectin levels in patients with ulcerative colitis at high risk of a relapse: a prospective, randomized, controlled study. United European Gastroenterol J 2015;3:72-9.

25 Sherlock ME, MacDonald JK, Griffiths AM, et al. Oral budesonide for induction of remission in ulcerative colitis. Cochrane Database Syst Rev 2015:CD007698.

26 Zhao X, Zhou C, Ma J, et al. Efficacy and safety of rectal 5 -aminosalicylic acid versus corticosteroids in active distal ulcerative colitis: a systematic review and network meta-analysis. Sci Rep 2017:7:46693.

27 BNF. National Institute for health and care excellence, 2021. Available: https://bnf.nice.org.uk/ [Accessed Jan 2022]. 
28 NHS England. National Cost Collection: National Schedule of NHS costs - Year 2019-20. Available: https://www.england.nhs.uk/ national-cost-collection/ [Accessed Jan 2022]

29 Curtis LA, Burns A. Unit Costs of Health \& Social Care 2020. PSSRU, University of Kent, 2020.

30 Point-Of-Care and home faecal calprotectin tests for monitoring treatment response in inflammatory bowel disease (MIB132). National Institute for health and care excellence. Available: www. nice.org.uk/guidance/mib132 [Accessed Jan 2022]

31 Waljee AK, Rogers MAM, Lin P, et al. Short term use of oral corticosteroids and related harms among adults in the United States: population based cohort study. BMJ 2017;357:j1415.

32 Sandborn WJ, Feagan BG, Marano C, et al. Subcutaneous golimumab induces clinical response and remission in patients with moderate-to-severe ulcerative colitis. Gastroenterology 2014;146:85-95.
33 Rutgeerts P, Sandborn WJ, Feagan BG, et al. Infliximab for induction and maintenance therapy for ulcerative colitis. $N$ Engl J Med 2005;353:2462-76.

34 EPAR. Adalimumab: EMEA/H/C/000481/II/0082. EMA, 2012. Available: https://www.ema.europa.eu/en/medicines/human/EPAR/ humira [Accessed Jan 2022].

35 Ruiz-Casas L, Evans J, Rose A, et al. The lucid study: living with ulcerative colitis; identifying the socioeconomic burden in Europe. BMC Gastroenterol 2021;21:456.

36 ClinicalTrials.gov. Study to assess effectiveness of therapy timely adjustment based on self-monitoring in patients suffering from mildto-moderate ulcerative colitis (OPTIMISE study). ClinicalTrials.gov: NCT04340895, 2019.

37 Mao R, Xiao Y-lian, Gao X, et al. Fecal calprotectin in predicting relapse of inflammatory bowel diseases: a meta-analysis of prospective studies. Inflamm Bowel Dis 2012;18:1894-9. 